Präv Gesundheitsf 2020 · 15:236-241 https://doi.org/10.1007/s11553-020-00760-6 Eingegangen: 8. November 2019

Angenommen: 3. Januar 2020

Online publiziert: 24. Januar 2020

(c) Der/die Autor(en) 2020

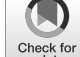

Stephanie Karg $(D)$ - Beate Blättner · Kerstin Krüger · Naomi Micheew

Fachbereich Pflege und Gesundheit, Hochschule Fulda, Fulda, Deutschland

\title{
Kompetenzen für Tätigkeiten in der Gesundheitsförderung
}

\section{Sichtweisen von Stakeholdern}

\begin{abstract}
Gesundheitsförderung ist kein geregelter Beruf des Gesundheitswesens, für den mit staatlichen Abschlussprüfungen der Nachweis der Befähigung erfolgt. Für Absolventinnen und Absolventen entsprechender Studiengänge ist es nicht immer einfach, potentiellen Arbeitgebern nachzuweisen, dass sie über die benötigten Kompetenzen verfügen; für Studierende nicht einfach zu wissen, auf welchen Kompetenzerwerb sie besonders achten müssen. Bislang fehlt weitgehend eine empirische Basis für entsprechende Beratung.
\end{abstract}

\section{Hintergrund und Fragestellung}

Mit der Einführung des Präventionsgesetzes in Deutschland im Jahr 2015 ist der Arbeitsmarkt für Absolventinnen und Absolventen von Studiengängen der Gesundheitsförderung breiter und differenzierter geworden. Mit 32 Bachelorund 32 Master-Studiengängen, die nach Angaben des Hochschulkompasses [6] "Gesundheitsförderung" im Titel tragen, ist das Ausbildungsangebot für solche Arbeitsplätze vielfältig. Der Abschlusstitel ist allerdings nicht geschützt. Studiengänge verschiedener Standorte können gleichermaßen "Gesundheitsförderung“ heißen, aber dennoch im Detail auf unterschiedliche Tätigkeiten vorbereiten. Auch der Abschlussgrad (Bachelor of Sciences oder Bachelor of Arts) gibt keine klare Orientierung. Studiengänge mit anderen Bezeichnungen (z. B. Gesundheitskommunikation, Public Health oder Gesundheitsmanagement) können umgekehrt mit vergleichbaren Inhalten für eine Tätigkeit in der Gesundheitsförde- rung qualifizieren. In der Akkreditierung der Studiengänge wird die generelle Berufsbefähigung überprüft, nicht aber die konkrete Eignung der vermittelten Kompetenzen für den jeweiligen Beruf [3].

Die International Union for Health Promotion and Education (IUPHE) hat sich, gefördert von der EU-Kommission, in den Jahren 2009 bis 2012 mit der Entwicklung eines Kompetenzrahmens für Gesundheitsförderung in Europa befasst, dem neben Literaturrecherchen und Abstimmungsprozessen beteiligter Akteure auch Befragungen von Praxiseinrichtungen zugrunde liegen [1]. Dieser Kompetenzrahmen ist unverbindlich. Er kann in der Entwicklung von Studiengängen einen groben Orientierungsrahmen geben; gibt Studierenden aber keine Antwort darauf, weshalb bestimmte Module für die Berufspraxis relevant sind, was sie während des Studiums tun können, um ihre Chancen auf einen Berufseinstieg nach dem Studium zu verbessern und ob ein Master-Studium nach dem BachelorAbschluss zwingend notwendig ist.

Ergebnis einer nicht veröffentlichten Verbleibstudie war, dass einige Absolventinnen und Absolventen eines Studiengangs Gesundheitsförderung ungenaue Vorstellungen über Beschäftigungsmöglichkeiten und die eigenen Kompetenzen als Hemmnis im Bewerbungsverfahren formulierten [9]. Ziel eines in den Jahren 2017 bis 2019 durchgeführten Projekts war es deshalb, Studierende darin besser zu unterstützen, bereits während des Studiums realistisch einschätzen zu lernen, in welchen Arbeitsfeldern sie nach Abschluss des Studiums arbeiten können, welche Kompetenzen sie dafür benötigen und wie sie die Chancen für einen Berufseinstieg in ihrem Wunschberufsfeld (z. B. durch die Wahl von Schwerpunkten und Praktika) verbessern können.

Grundlage dieser Unterstützung sollten Interviews mit Stakeholdern sein, die primär der Frage nachgehen, welche Kompetenzen in einzelnen Arbeitsfeldern benötigt werden. Sekundär interessierte, welche Tätigkeitsbereiche für Absolvierende eines Bachelor-Studienganges Gesundheitsförderung geeignet sind und wie sich Studierende während des Studiums gezielt auf den Berufseinstieg vorbereiten können. Aus den Ergebnissen sollten Qualifikationspfade entwickelt werden, die der Beratung der Studierenden dienen.

\section{Methode und Material}

In einem ersten Schritt wurde auf der Basis von aktuellen Stellenausschreibungen eine grobe Übersicht über ausgeschriebene Arbeitsbereiche und Tätigkeitsinhalte erstellt. Identifizierte Tätigkeitsfelder wurden nach der Analyse der Interviews neu zu Gruppen zusammengefasst.

In zwei Erhebungswellen (Januar bis Mai 2018 und November 2018 bis März 2019) wurden 28 Interviews mit Stakeholdern durchgeführt. Als Stakeholder wurden Personen definiert, die entweder im jeweiligen Tätigkeitsfeld selbst beschäftigt sind oder als Personalverantwortliche oder Geschäftsführer für entsprechende Arbeitsfelder fungieren. Dabei wurden sowohl Berufseinsteigerinnen und -einsteiger als auch erfahrene Berufstätige gewählt. Berücksichtigt werden sollten weiterhin Institutionen, die regional, landes- oder bundesweit tä- 


\begin{tabular}{l|ll}
$\begin{array}{l}\text { Tab. } 1 \text { Übersicht der befragten Institutionen } \\
\text { Staatliche Institutionen }\end{array}$ & $\begin{array}{l}\text { Öffentlich-rechtliche } \\
\text { Körperschaften }\end{array}$ & Freie Träger, Vereine, Unternehmen \\
\hline $\begin{array}{l}\text { Ministerien für Gesund- } \\
\text { heit }\end{array}$ & $\begin{array}{l}\text { Berufsgenossenschaften } \\
1 \text { Interview }\end{array}$ & $\begin{array}{l}\text { Landesvereinigungen für Gesundheits- } \\
\text { förderung }\end{array}$ \\
$\begin{array}{l}\text { Öffentliche } \\
\text { Gesundheitsdienste } \\
6 \text { Interviews }\end{array}$ & $\begin{array}{l}\text { Gesetzliche } \\
\text { Krankenversicherungen in 3 Einrichtungen } \\
5 \text { Interviews }\end{array}$ & $\begin{array}{l}\text { Präventionsfachstellen } \\
\text { 3 Interviews } \\
\text { Unternehmen, Betriebe } \\
4 \text { Interviews }\end{array}$ \\
\hline
\end{tabular}

tig sind, Körperschaften des öffentlichen Rechts, private Unternehmen und öffentliche Einrichtungen oder ihnen nahestehende Verbände, die in der Gesundheitsförderung von Relevanz sind (• Tab. 1).

Der Interviewleitfaden wurde nach dem Prinzip des Sammelns, Prüfens, Sortierens und Subsumierens entwickelt und enthält erzählgenerierende Einstiegsfragen, konkrete Nachfragen und Steuerungsfragen [5]. Gefragt wurde nach Tätigkeiten, notwendigen Kompetenzen, Anforderungen der Tätigkeit, Erwartungen an Stelleninhaberinnen und -inhaber sowie Empfehlungen für Studierende zum Berufseinstieg. Die Einstiegsfrage wurde erzählgenerierend gestellt und bezog sich auf den Arbeitsalltag. In der Formulierung erfolgte eine Anpassung je nach beruflichen Hintergrund und Position in der Hierarchieebene der Befragten.

Durchgeführt wurden die Interviews von acht dafür speziell angeleiteten Studierenden in einem studentischen Projekt und der koordinierenden Mitarbeiterin. Nach jedem Interview wurde auf Basis der Tonbandaufzeichnung und der handschriftlichen Notizen ein Interviewprotokoll angefertigt.

Die Auswertung der Interviews erfolgte in einem Verfahren des Fallvergleichs, der Fallkontrastierung und der Typenbildung, die auf der Bildung von Kategorien und Subkategorien sowie von Strukturhypothesen beruht [8]. Die Auswertung erfolgte in einem diskursiven Prozess, an dem mehrere Personen beteiligt waren.

\section{Ergebnisse}

\section{Charakterisierung der Arbeitsfelder}

Identifiziert wurden die Arbeitsfelder betriebliche Gesundheitsförderung (BGF), Gesundheitsberichterstattung, Gesundheitskommunikation sowie Koordinations- und Entwicklungsarbeit.

Aufgaben der Koordinations- und Entwicklungsarbeit wurden im Dachsetting Kommune und bei koordinierenden Landeseinrichtungen identifiziert; sie sind auch z. B. bei Sozialversicherungen denkbar. Eigene Arbeitsfelder in den nichtbetrieblichen Settings konnten außerhalb solcher Tätigkeiten nicht identifiziert werden. Zwei Ausrichtungen konnten unterschieden werden: die Koordinations- und Netzwerkarbeit einerseits und die Projektarbeit andererseits. Während sich die Projektarbeit adressaten- und ggf. themenspezifisch an der Logik des „Public Health Action Cycles" $[7,11]$ orientiert, konzentriert sich die Netzwerkarbeit auf die Vernetzung von Akteuren, der Bildung und Begleitung von runden Tischen und der Verbreitung des Themas Gesundheitsförderung auf kommunaler oder Landesebene. Die Arbeitsfelder sind aber nicht trennscharf. So können beide Aufgaben einer Person zugeordnet sein oder im Laufe der Beschäftigungsdauer variieren.

In der BGF konnten Unternehmen, Krankenkassen und Dienstleistungsunternehmen in die Analyse einbezogen werden. Unterschiede ergeben sich weniger aus den Arbeitsinhalten, sondern aus dem Grad der bestehenden Umsetzung des BGF und den sich daraus entwickelnden Routinen. BGF weist Merkmale der Projekt- und der Netzwerkarbeit auf. Anders als bei nichtbetrieblichen Settings haben sich hier aber eigene Arbeitsfelder in einer spezifischen Spezialisierung entwickelt.

Gesundheitsberichterstattung ist relevant für Kommunen, Länder und den Bund, aber auch in großen institutionellen Settings wie der BGF. Die Tätigkeiten sind vergleichbar, jedoch unterscheiden sich die Datenlage, Datenmenge sowie die Themen und die Möglichkeiten, auf statistische Aufbereitungen der Daten zurückgreifen zu können. Die Präsentation der Daten für den jeweiligen Adressatenkreis weist dabei Überschneidungen zu Fragen auf, die sich in der Gesundheitskommunikation stellen.

Gesundheitskommunikation lässt sich als Querschnittsthema beschreiben, das in jedem Arbeitsfeld Relevanz hat. Spezifische Kompetenzen werden z.B. dann benötigt, wenn es um die systematische Entwicklung und Koordination von Gesundheitskampagnen geht, die sich an die Bevölkerung oder Subgruppen der Bevölkerung richten.

\section{Übergreifende und spezifische Arbeitsschwerpunkte}

Quer zu den identifizierten Arbeitsbereichen stehen fünf Arbeitsschwerpunkte (- Tab.2), die in unterschiedlicher Intensität in den meisten Arbeitsfeldern relevant sind.

Projektmanagement beinhaltet die Konzeption, Umsetzung, Begleitung und ggf. Evaluation von Projekten. Dies kann die Beantragung, Kalkulation, Budgetverwaltung und das Controlling der Projektmittel einschließen. Projektmanagement ist der herausragende Schwerpunkt im Typ „Projektarbeit“ im Arbeitsfeld Koordinations- und Entwicklungsarbeit. Er ist auch in der BGF, der Gesundheitsberichterstattung und der Gesundheitskommunikation relevant, weil dort jeweils Projekte umgesetzt werden.

Netzwerkarbeit beinhaltet den Aufbau und Koordination von Netzwerken sowie die Beteiligung an Netzwerken und Kooperationen. Dazu zählen die Organisation und Durchführung von Netzwerktreffen, die Leitung der Treffen und ggf. die Beratung der beteiligten Institutionen und Personen. Koordinationstätig- 
keiten umfassen darüber hinaus Tätigkeiten, die sich auf die Zusammenarbeit mit Projektpartnern, Agenturen oder weiteren Dienstleistern beziehen, um gemeinsam Konzepte, Kampagnen und Projekte umzusetzen. Entsprechende Tätigkeiten sind in unterschiedlichen Ausprägungen in allen Arbeitsfeldern relevant.

Konzeptionelles Arbeiten umfasst die Entwicklung und Durchführung von Konzepten wie beispielsweise von Workshops, Informationsveranstaltungen und Fachtagungen, von Gesundheitskampagnen, gesundheitsfördernden Projekten und Maßnahmen. Konzeptionelles Arbeiten ist in allen Arbeitsfeldern gefragt. Was sich unterscheidet ist lediglich der Gegenstand der konzipiert wird.

Wissenschaftliches Arbeiten ist eng mit dem konzeptionellen Arbeiten verbunden und beinhaltet die Erfassung von Studienlagen zum bearbeitenden Thema, die Durchführung von Literaturrecherchen, Bedarfsanalysen und eigenen Erhebungen, die Auswertung solcher Daten, die Aufbereitung der Ergebnisse und das Verfassen von wissenschaftlichen Texten und Publikationen sowie die Vorstellung der Ergebnisse z. B. auf Kongressen. In der Gesundheitsberichterstattung und der Projektarbeit ist dies besonders relevant, aber auch im BGF und der Gesundheitskommunikation.

Unter Öffentlichkeitsarbeit sind alle Tätigkeiten $\mathrm{zu}$ verstehen, die der Informationsverbreitung von Projekten, Kampagnen, Konzepten und wissenschaftlichen Ergebnissen dienen. Dabei erfolgt die Erstellung von Pressemitteilungen, Flyern, Broschüren und Stellungnahmen, die Gestaltung von Intra- und Internetseiten und Broschüren sowie die Präsenz und Vorträge auf Fachtagungen, Kongressen und bei Veranstaltungen. Öffentlichkeitsarbeit ist in allen Arbeitsfeldern relevant, in der Gesundheitskommunikation ein besonderer Schwerpunkt.

Für arbeitsfelderspezifische Aufgaben sind insbesondere die Anwendung spezifischer Methoden des jeweiligen Arbeitsfeldes von Bedeutung. In der BGF sind dies beispielsweise die Beratung von Firmen und deren Führungskräften, die Durchführung von Gesundheitszirkeln oder Gefährdungsbeurteilungen

Präv Gesundheitsf 2020 • 15:236-241 https://doi.org/10.1007/s11553-020-00760-6

(c) Der/die Autor(en) 2020

\section{S. Karg · B. Blättner · K. Krüger · N. Micheew}

\section{Kompetenzen für Tätigkeiten in der Gesundheitsförderung. Sichtweisen von Stakeholdern}

\section{Zusammenfassung}

Hintergrund. Gesundheitsförderer werden am Arbeitsmarkt gebraucht. Für Absolventinnen und Absolventen entsprechender Studiengänge scheint aber die Orientierung nicht leicht zu sein, welche Kompetenzen relevant sind und am Arbeitsmarkt erwartet werden.

Fragestellung. Es interessierte, welche Kompetenzen aus Sicht von Stakeholdern benötigt werden und wie Studierende sich während des Studiums gezielt auf eine Erwerbstätigkeit vorbereiten können. Material und Methode. Es wurden leitfadengestützte Interviews mit 28 Stakeholdern geführt und mittels Kategorisierung und Typenbildung ausgewertet. Als Stakeholder wurden Personen definiert, die im jeweiligen Tätigkeitsfeld beschäftigt waren oder als Personalverantwortliche bzw. Geschäftsführer für entsprechende Arbeitsfelder fungierten.
Ergebnisse. Die Bereiche Betriebliche Gesundheitsförderung, Gesundheitsberichterstattung, Gesundheitskommunikation und Koordinations- und Entwicklungsarbeit sind wesentliche Arbeitsfelder. In den benötigten Kompetenzen und Aufgabengebieten weisen sie eher Gemeinsamkeiten als Unterschiede auf. Persönliche und soziale Kompetenzen können über die Einstellung entscheiden. Schlussfolgerungen. Die Förderung persönlicher und sozialer sowie eher generalistischer Kompetenzen in der Gesundheitsförderung sollte im Studium einen Schwerpunkt darstellen. Daneben sollten Studierende individuelle Kompetenzprofile entwickeln.

Schlüsselwörter

Professionelle Kompetenzen · Arbeitsplatz - Stakeholder Analyse - Studium . Gesundheitsförderung

\section{Competences for working in health promotion. Perceptions of stakeholders}

\section{Abstract}

Background. Health promoters are essential in the job market. It appears to be difficult for graduates to comprehend which skills are relevant and which are expected in the jobmarket.

Objectives. It was of interest, which competences are needed from point of view of different stakeholders and how students can prepare for the employment during their studies.

Methods. In total, 28 qualitative interviews have been conducted with stakeholders from health promotion and have been analysed by categorisation and typification.Stakeholders are defining as individuals who are working in different fields within health promotion, as a personnel officer or as a general manager. Results. Occupational health promotion, health monitoring, health communication and coordination and development work are fundamental fields of job placement. There are more similarities than differences in the required competences. Personal and social competences are necessary and can be vital for being employed.

Conclusions. The promotion of personal and social skills as well as generalised competences in health promotion should be a focus during health promotion studies. Moreover, the students should develop their own profile of competences.

\section{Keywords}

Professional Competence - Workplace . Stakeholder Analysis · StudyProgram · Health Promotion oder von Verfahren des Betrieblichen Eingliederungsmanagements sowie die Organisation von Gesundheitstagen. In der Gesundheitsberichterstattung geht es beispielsweise um die Aufbereitung statistischer Daten für spezielle
Adressatengruppen, zu denen vor allem Entscheidungsträger gehören.

\section{Relevante Kompetenzen}

Berufliche Handlungskompetenz lässt sich in Fachkompetenz, Methodenkom- 


\begin{tabular}{|c|c|c|c|c|c|}
\hline \multirow[t]{2}{*}{ Schwerpunkte } & \multirow{2}{*}{$\begin{array}{l}\text { Betriebliche } \\
\text { Gesundheits- } \\
\text { förderung }\end{array}$} & \multicolumn{2}{|c|}{ Koordinations- und Entwicklungsarbeit } & \multirow{2}{*}{$\begin{array}{l}\text { Gesundheits- } \\
\text { bericht- } \\
\text { erstattung }\end{array}$} & \multirow{2}{*}{$\begin{array}{l}\text { Gesundheitskommunikation } \\
\text { Koordination von Ge- } \\
\text { sundheitskampagnen }\end{array}$} \\
\hline & & Projektarbeit & $\begin{array}{l}\text { Koordinations- und } \\
\text { Netzwerkarbeit }\end{array}$ & & \\
\hline Projektmanagement & (1) & $\bullet$ & D & (1) & (1) \\
\hline $\begin{array}{l}\text { Netzwerkarbeit, Koordinations- } \\
\text { tätigkeiten }\end{array}$ & - & - & $\bullet$ & D & D \\
\hline Konzeptionelles Arbeiten & $\bullet$ & $\bullet$ & - & $\bullet$ & $\bullet$ \\
\hline Wissenschaftliches Arbeiten & - & $\bullet$ & O & $\bullet$ & D \\
\hline Öffentlichkeitsarbeit & D & - & D & - & $\bullet$ \\
\hline \multicolumn{6}{|c|}{ O kaum relevant, relevant, besonders relevant } \\
\hline
\end{tabular}

petenz, Sozialkompetenz und Selbstkompetenzen gliedern [10, vgl. 4]. Sozial- und Selbstkompetenz sind in den Arbeitsfeldern der Gesundheitsförderung von übergeordneter Bedeutung, während die Fach- und Methodenkompetenzen sich in den Arbeitsfeldern am ehesten voneinander unterscheiden.

$\mathrm{Zu}$ den in den Interviews genannten Selbstkompetenzen gehören souveränes Auftreten, Engagement, eigenverantwortliches Handeln und eine selbstständige Arbeitsweise, Flexibilität, gutes Zeitmanagement und Durchhaltevermögen. Als besonders wichtig wird die Sozialkompetenz bezeichnet, die v. a. kommunikative Fähigkeiten, Teamfähigkeit und Konfliktbewältigungskompetenzen umfasst. Methodenkompetenzen beziehen sich auf Methoden des wissenschaftlichen Arbeitens und des Projektmanagements sowie Moderations- und Präsentationstechniken. Fachlich ist ein fundiertes Wissen über Gesundheitsförderung gefordert, daneben aber je nach Arbeitsfeld auch adressatenspezifische und institutionelle Kenntnisse.

Eine Differenzierung nach Kompetenzniveaus, also etwa Niveau 6 oder 7 des Deutschen Qualifikationsrahmens [4], wird aus den Interviews nicht deutlich. Generell lässt sich aber sagen: Ein Berufseinstieg nach dem Bachelor-Abschluss ist möglich, aufgrund der besseren Akzeptanz bei Kooperationspartnern wird der zusätzliche Erwerb eines MasterAbschlusses häufiger empfohlen. Dies ist von der regionalen Reichweite des Arbeitsfeldes, insbesondere aber vom Grad der Verantwortungsübernahme auf der jeweiligen Position abhängig. Werden leitende Funktionen angestrebt, ist ein Masterabschluss Voraussetzung.

\section{Strategien zur Vorbereitung auf den Berufseinstieg}

Potenzielle Arbeitgeber interessieren sich nicht nur für den Studienabschluss und die dort erreichten Leistungsbewertungen, sondern immer auch für praktische Erfahrungen, die während oder nach dem Studium erworben wurden. Die Auswahl der Praktikumsstelle und die Leistungen im Praktikum haben deshalb hohe Relevanz für das Gelingen des Berufseinstiegs. Praxisphasen bieten zudem die Chance, Kontakte zu knüpfen, zu pflegen sowie Netzwerke aufzubauen. Von Vorteil kann es sein, über das Praktikum hinaus Kontakt zu der Praktikumseinrichtung zu halten, um u.a. über freiwerdende Stellen informiert zu werden.

Als weitere praktische Erfahrungen werden zusätzliches Engagement durch die Beteiligung an Hochschulaktivitäten, wie z.B. Tutoring oder die Mitarbeit in Forschungsprojekten, durch ehrenamtliche Tätigkeiten oder durch nebenberufliche Arbeit gewürdigt. Dies unterscheidet Bewerberinnen und Bewerbern voneinander und hilft ggf. adressatenspezifische oder institutionelle Kompetenzen nachzuweisen.

Die Erarbeitung eines individuellen Kompetenzprofils kann für den Berufseinstieg relevant sein. Eine zu enge Festlegung auf ein bestimmtes Feld kann zugleich hinderlich sein, wenn nicht auch Flexibilität gezeigt werden kann. Es geht primär darum, die Kernkompetenzen unter Beweis zu stellen, die den übergreifenden Arbeitsschwerpunkten zugeordnet sind.

Die Zeit des Studiums sollte für eine individuelle Weiterbildung genutzt wer- den, indem z. B. Gastvorträge, Tagungen oder Kongresse besucht werden sowie eigene inhaltlichen Interessen nachgegangen wird. Unterschiedliche Themen, z. B. in Prüfungsleistungen, zu bearbeiten hilft, einen Überblick über die vielfältigen Inhalte und Ausrichtungen zu erreichen. Dies steht nicht im Gegensatz zur Fokussierung auf ein eigenes Profil. Die Gradwanderung zwischen Breite und Tiefe der Ausbildung kann für die Einstellung entscheidend sein. Die gesammelten Erfahrungen sollten reflektiert werden und bei der Bewerbung für Arbeitsstellen differenziert dargestellt werden.

Gerade bei der Vorbereitung auf die berufliche Zukunft und einen erfolgreichen Start in das Berufsleben sollte eine entsprechende Eigeninitiative der Studierenden erfolgen und die Verantwortung dafür auch selbstständig getragen werden.

\section{Qualifikationspfade}

Ein Qualifikationspfad beschreibt, über welche Wege ein bestimmtes Qualifikationsziel erreicht werden kann. Bezogen aufStudiengänge sind Qualifikationspfade Beschreibungen, wie Wahloptionen und Module im Studium so genutzt werden können, dass ein individuelles Kompetenzprofil entsteht. Qualifikationspfade sollten Auskunft über spezifische Kompetenzen und deren möglichen Erwerb geben. Darüber hinaus bilden sie den Studienverlauf mit den verschiedenen Wahlmöglichkeiten ab.

Die $\bullet$ Tab. 3 zeigt exemplarisch einen der entwickelten Qualifikationspfade. Dieser beinhaltet alle notwendigen Informationen zu notwendigen Kompetenzen und zur Ausrichtung des Studiums, 
Tab. 3 Exemplarische Darstellung eines Qualifikationspfades

\section{Qualifikationspfad Projektarbeit \\ Angestrebte Arbeits- Mitarbeit in oder Leitung von Projekten \\ felder}

Besondere Kompetenzen

Fachkompetenz Kenntnisse der jeweiligen Arbeitsfelder und der Adressatengruppen; gute Fachkenntnisse der Strategien der Gesundheitsförderung

Methodenkompetenz Anwendung von Kenntnissen der empirischen Sozialforschung (in der Bedarfsanalyse und ggf. der Evaluation); Projektmanagement, ggf. Antragserfahrung

Sozialkompetenz Zugang zu Zielgruppen, Empathie; Kooperationsfähigkeit

Persönliche Kompetenzen

Selbstständiges Arbeiten; Lernfähigkeit; sicheres Auftreten; guter Schreibstil; Präsentationsfähigkeit

Sprachkenntnisse

Lesekompetenz englischsprachiger Fachliteratur; ggf. können Sprachkenntnisse der Adressatengruppe von Vorteil sein

Notwendiger $A b$ schluss

Einstieg nach Bachelorabschluss möglich; (berufsbegleitendes) Masterstudium z. B. in Public Health für Leitung von Projekten sinnvoll

Vorbereitung im Studium

\begin{tabular}{|c|c|}
\hline $\begin{array}{l}\text { Besonders relevan- } \\
\text { te Module (außer } \\
\text { Schwerpunkt) }\end{array}$ & $\begin{array}{l}\text { Gesundheitswissenschaftliches Arbeiten und Denken, } \\
\text { Handlungsfelder der Prävention, } \\
\text { Strategien der Gesundheitsförderung, } \\
\text { empirische Sozialforschung, } \\
\text { ggf. Evidenzbasierung oder Evaluationsmethoden }\end{array}$ \\
\hline Studienbegleitend & $\begin{array}{l}\text { Mitarbeit in Projekten in und außerhalb der Hochschule, ggf. ehren- } \\
\text { amtliches Engagement; wissenschaftliches Schreiben trainieren; aktiver } \\
\text { Besuch von Kongressen (Armut und Gesundheit) und Tagungen }\end{array}$ \\
\hline Wahlmodul & Projektmanagement \\
\hline Praktikum & $\begin{array}{l}\text { Projekte der Gesundheitsförderung unabhängig vom Träger; aktive } \\
\text { Gestaltung des Praktikums; nach dem Praktikum aktiv Kontakt halten, } \\
\text { Abschlussarbeit anstreben }\end{array}$ \\
\hline Schwerpunkt & $\begin{array}{l}\text { Gesundheitsförderung in institutionellen Settings; Gesundheitsförde- } \\
\text { rung in Kommunen }\end{array}$ \\
\hline $\begin{array}{l}\text { Projekt- und Themen- } \\
\text { wahl }\end{array}$ & $\begin{array}{l}\text { Ggf. Themen und Adressatengruppen in relevantem Bereich fokussieren; } \\
\text { konkrete Projektkonzeption und Durchführung }\end{array}$ \\
\hline Abschlussarbeit & Sollte aus dem Praktikum hervorgehen \\
\hline
\end{tabular}

wenn eine Projektarbeit angestrebt wird. Es wird verdeutlicht, welche Elemente des Studiums hier besonders relevant sind und wie während des Studiums praktische Erfahrungen gesammelt werden können, die beim Berufseinstieg unterstützen können. Qualifikationspfade werden in der Beratung durch das Praxisreferat genutzt.

\section{Diskussion}

Die Breite der Institutionen, in denen Interviews durchgeführt werden, weist Lücken auf. Die Dichte neuer Erkenntnisse in der Analyse der Interviews ließ aber eine theoretische Sättigung vermuten, die gegen die Erhebung weiterer Daten sprach. nicht schlüssig erscheinen. in der Berufspraxis der Gesundheitsförderung zu unterscheiden. Studiengänge sollten sich darauf konzentrieren, die generischen Kompetenzen zu vermitteln und Studierenden die Chancen geben, über die Wahl spezifischer Kompetenzen ihr individuelles Berufsprofil zu entwickeln. Dieses spezifische Profil wird dann relevant, wenn die generischen Kompetenzen nachgewiesen werden können.

Die Differenzierung nach persönlichen, sozialen, fachlichen und methodischen Kompetenzen folgt der Logik handlungsorientierter Didaktik. Sie ist nicht die einzige Möglichkeit einer Differenzierung. So folgt die Gliederung des CompHP-Kompetenzrahmens [1] eher entlang der Logik der Ottawa-Charta [12], daraus lassen sich allenfalls indirekt persönliche oder soziale Kompetenzen ableiten. Der Fachqualifikationsrahmen Gesundheitsförderung, der von einer Expertengruppe deutscher Hochschulen erstellt wurde, ordnet Kompetenzen anhand der Dublin-Deskriptoren und des „Public Health Action Cycles" ein [2]. Solche Qualifikationsrahmen werden vornehmlich dazu verwendet, die Curricula der Studiengänge und die entsprechenden Module inhaltlich zu gestalten. Eine Vergleichbarkeit von Studiengängen könnte damit hergestellt werden. Inwieweit sich Studierende daran orientieren können, bleibt aber fraglich.

Mithilfe der im Projekt entwickelten Qualifikationspfade wurde alternativ eine Möglichkeit geschaffen, neben fachlichen Kompetenzen auch methodische, soziale und persönliche Kompetenzen darzustellen, die aus Sicht der Stakeholder in der Praxis der Gesundheitsförderung relevant sind.

\section{Fazit für die Praxis}

heitsförderung inzwischen z. B. mit der Darstellung von Präventionsketten über den reinen Settings-Ansatz hinaus. Arbeitsfelder könnten auch nach Schwerpunktaufgaben differenziert werden. Die Ergebnisse der Analyse ließen dies aber

Abgeleitet aus der hohen Relevanz übergreifender Arbeitsschwerpunkte scheint es relevant, zwischen generischen und spezifischen Kompetenzen

\section{- Berufsperspektiven und relevante Kompetenten zu kennen, könnte Studierende auf den Berufseinstieg besser vorbereiten. Qualifikations- pfade, die aufzeigen, wie Wahlop- tionen für die Profilbildung sinnvoll genutzt werden können, können der Orientierung dienen. \\ - Eher generalistische Kompetenzen in der Gesundheitsförderung domi-}




\section{nieren gegenüber sehr spezifischen Kompetenzen für einzelne Tätigkeits- felder. \\ - Neben den fachlichen und metho- dischen Kompetenzen sollte die Entwicklung persönlicher und so- zialer Kompetenzen im Studium unterstützt werden, da diese für den Berufseinstieg ausschlaggebend sein können.}

\section{Korrespondenzadresse}

\section{Stephanie Karg}

Fachbereich Pflege und Gesundheit, Hochschule Fulda

Leipziger Straße 123, 36037 Fulda, Deutschland stephanie.karg@pg.hs-fulda.de

Funding. Open Access funding provided by Projekt DEAL.

\section{Einhaltung ethischer Richtlinien}

Interessenkonflikt. S. Karg, B. Blättner, K. Krüger und N. Micheew geben an, dass kein Interessenkonflikt besteht.

Ein Votum der Ethikkommission wurde nicht eingeholt, da ausschließlich Erwachsene im vollen Besitz ihrer kognitiven Fähigkeiten auf freiwilliger Basis interviewt wurden. Die Interviews wurden nach Aufklärung und schriftlicher Zustimmung der befragten Personen durchgeführt. Alle Anforderungen des Datenschutzes wurden eingehalten.

Open Access Dieser Artikel wird unter der Creative Commons Namensnennung 4.0 International Lizenz veröffentlicht, welche die Nutzung, Vervielfältigung, Bearbeitung, Verbreitung und Wiedergabe in jeglichem Medium und Format erlaubt, sofern Sie den/die ursprünglichen Autor(en) und die Quelle ordnungsgemäß nennen, einen Link zur Creative Commons Lizenz beifügen und angeben, ob Änderungen vorgenommen wurden.

Die in diesem Artikel enthaltenen Bilder und sonstiges Drittmaterial unterliegen ebenfalls der genannten Creative Commons Lizenz, sofern sich aus der Abbildungslegende nichts anderes ergibt. Sofern das betreffende Material nicht unter der genannten Creative Commons Lizenz steht und die betreffende Handlung nicht nach gesetzlichen Vorschriften erlaubt ist, ist für die oben aufgeführten Weiterverwendungen des Materials die Einwilligung des jeweiligen Rechteinhabers einzuholen.

Weitere Details zur Lizenz entnehmen Sie bitte der Lizenzinformation auf http://creativecommons.org/ licenses/by/4.0/deed.de.

\section{Literatur}

1. Barry MM, Battel-Kirk B, Davison $\mathrm{H}$, Dempsey C, Parish R, Schipperen M, ZilnykA (2014) Das CompHP-
Rahmenkonzept für die Gesundheitsförderung Kernkompetenzen - Professionelle Standards Akkreditierung, Deutsche Kurzfassung. BZgA, Köln

2. Baumgarten K, Blättner B, Dadaczynski K, Hartmann T (2015) Entwicklung eines Fachqualifikationsrahmens für die Studienbereiche Gesundheitswissenschaften/Public Health und Gesundheitsförderung in Deutschland. Präv Gesundheitsf 10:320-327

3. Blättner B, Hartmann T, Baumgarten K (2019) Aus- und Weiterbildung in Gesundheitsförderung und Prävention. www.leitbegriffe. bzga.de/alphabetisches-verzeichnis/aus-undweiterbildung-in-gesundheitsfoerderung-undpraevention/. Zugegriffen: 29. Juli 2019 (BZgA (Hrsg.): Leitbegriffe der Gesundheitsförderung)

4. Bundesministerium für Bildung und Forschung, Sekretariat der Kultusministerkonferenz (Hrsg) (2019) Wie ist der DQR aufgebaut? https://www. dqr.de/content/2314.php. Zugegriffen: 2. Aug. 2019

5. Helfferich C (2011) Die Qualität qualitativer Daten. Manual für die Durchführung qualitativer Interviews, 2. Aufl. Springer, Wiesbaden

6. Hochschulrektorenkonferenz (o.J.) Hochschulkompass. https://www.hochschulkompass.de. Zugegriffen: 4.Okt. 2019

7. Institute of Medicine (1988) The future of public health. National Academy Press, Washington

8. Kelle U, Kluge S (2010) Vom Einzelfall zum Typus. Fallvergleich und Fallkontrastierung in der qualitativen Sozialforschung, 2. Aufl. VS, Wiesbaden

9. Klärs G, Hergenhan L, Blättner B (2017) Absolventenverbleibstudie für den Bachelorstudiengang Gesundheitsförderung. Hochschule Fulda, Unveröffentlichtes Manuskript

10. Kultusministerkonferenz (Hrsg) (2018) Handreichung für die Erarbeitung von Rahmenlehrplänen der Kultusministerkonferenz für den berufsbezogenen Unterricht in der Berufsschule und ihre Abstimmung mit Ausbildungsordnungen des Bundes für anerkannte Ausbildungsberufe. Sekretariat der Kultusministerkonferenz, Berlin

11. Rosenbrock R, Hartung S (2011) Public Health Action Cycle/Gesundheitspolitischer Aktionszyklus. In: Bundeszentrale für gesundheitliche Aufklärung (BZgA) (Hrsg) Leitbegriffe der Prävention und Gesundheitsförderung. Glossar zu Konzepten und Methoden, 5. Aufl. Verlag für Gesundheitsförderung, Werbach-Gamburg, S469-471

12. Europa (1986) Ottawa-Charta zur Gesundheitsförderung. http://www.euro.who.int/de/ publications/policy-documents/ottawa-charterfor-health-promotion-1986. Zugegriffen: 1. Nov. 2019 